\title{
METODOLOGÍA PARA MONITOREAR RIESGOS ESTRATÉGICOS METHODOLOGY TO MONITOR STRATEGIC RISKS
}

\author{
METHODOLOGY TO MONITOR STRATEGIC RISKS
}

Rubi Consuelo Mejía Quijanoํㄹ ${ }^{1}$ Eduart Humberto Villanueva Herrera ${ }^{2}$
${ }^{1 .}$ Contadora Pública, Especialista en Auditoría de sistemas y en Gerencia de proyectos, Magister en Ciencias de la Administración de la Universidad EAFIT. Coordinadora del Grupo de investigación en Información y Gestión de la Universidad EAFIT y profesora del departamento de Contaduría Pública de la misma institución. Teléfono: (57-4) 2619500 Ext. 9248. E-mail: rmejia@eafit.edu.co
2. Administrador de empresas de la Universidad la Gran Colombia, estudiante de la Maestría en Administración MBA) de la Universidad EAFIT. Asistente de investigación y de proyectos de la Universidad EAFIT. Teléfono: (57-4) 2619500 Ext. 9959. E-mail: evillanu@eafit.edu.co

Recibido: 20 de Noviembre de 2014

Aceptado: 10 de Diciembre de 2014

Correspondencia: Rubi Consuelo Mejía Quijano, Grupo de investigación en Información y Gestión de la Universidad EAFIT Teléfono: (57-4) 2619500 Ext. 9248. E-mail: rmejia@eafit.edu.co

\section{RESUMEN}

El presente artículo expone los avances de la investigación Metodología para monitorear riesgos estratégicos, que realiza el Grupo de Investigación en Información y Gestión de la Universidad EAFIT. La investigación busca diseñar una metodología que permita monitorear diferentes variables del entorno, tanto interno como externo a las organizaciones, que pueden impedir el cumplimiento de sus objetivos estratégicos.

Este estudio es de carácter cualitativo, exploratorio y propositivo. Para cumplir el objetivo de la investigación se realizó una revisión documental acerca de las diferentes prácticas de monitoreo organizacional -interno y externo-, tales como la inteligencia económica, la inteligencia competitiva, las alertas tempranas, la inteligencia de mercado, el cuadro de mando integral, etc.; para reconocer instrumentos, herramientas y mecanismos que sirvieran de apoyo en la elaboración de la metodología. Adicionalmente se estudió la información sobre monitoreo en las normas y estándares internacionales de riesgos, para contrastar estos resultados con las diferentes prácticas de monitoreo que se utilizan en el mundo empresarial. La consulta de las prácticas de monitoreo se realizó a través de entrevistas abiertas y semiestructuradas.

Los resultados de esta investigación indican la necesidad de contar con una metodología que incorpore los prerrequisitos para su aplicación -sensibilización hacia la importancia de esta práctica, identificación, evaluación y priorización de riesgos-, e incluya además el establecimiento del mecanismo de monitoreo de los riesgos priorizados, la búsqueda de información, su validación, análisis, y finalmente la toma de decisiones, que permitan responder ante los riesgos estratégicos que pueden afectar las organizaciones.

Palabras claves: Riesgo, Riesgo estratégico, Monitoreo estratégico, Estrategia. 


\begin{abstract}
This paper presents the advances in the research Methodology to monitor strategic risks done by the Universidad EAFIT Research Group on Information and Management. The objective of the research is to design a methodology that allows monitoring different environment variables, both inside and outside the organizations, which can hinder the fulfillment of their strategic goals.

This is a qualitative and exploratory study An internal and external review of documents related to different practices of organizational monitoring such as economic intelligence, competitive intelligence, early warnings, market intelligence and balanced scorecard was made in order to identify the instruments and mechanisms used in the building up of the methodology. Additionally, we studied information on monitoring from the international risk standards to contrast these results with the different monitoring practices used in the business world. The consultation on the monitoring practices was done through open and semi-structured interviews.

The results of this research determine the need of having a methodology that incorporates the prerequisites for its implementation -sensitization on the importance of this practice, identification of the risks, evaluation and prioritization. That also includes the establishment of the information needs, the mechanism to monitor the prioritized risks, the search for information, its validation, analysis, and the decision making; that allow the organizations to respond to the strategic risks that can affect them.
\end{abstract}

Keywords: Risk, strategic risk, strategic monitoring, strategy.

\section{INTRODUCCIÓN}

El ambiente en el cual se desempeñan las empresas modernas tiene como premisa la incertidumbre, ocasionada por la globalización, los cambios constantes en las variables políticas, económicas, sociales, tecnológicas, ambientales y legales ${ }^{1}$, entre otros. En este entorno la toma de decisiones en las organizaciones es compleja, lo que incrementa los riesgos ${ }^{2}$ empresariales, y por ende la probabilidad de fracaso; por lo cual la administración de riesgos se convierte en una disciplina necesaria para preservar las organizaciones y protegerlas de pérdidas que afecten su estabilidad. (COSO, 2004)

Los riesgos que impiden el cumplimiento de los lineamientos estratégicos pueden generarse en la organización o provenir del entorno; para estar preparados para afrontarlos se necesitan mecanismos que permitan detectarlos a tiempo y hacerles seguimiento.

\footnotetext{
${ }^{1}$ Un análisis de estas variables es denominado Pestel. (Kaplan \& Norton, 2008:72).

${ }^{2}$ Comprende las etapas de identificación, calificación, evaluación, comunicación, monitoreo, diseño e implementación de medidas de tratamiento de los riesgos.
}

Por lo anterior, el Grupo de Investigación en Información y Gestión de la Universidad EAFIT ${ }^{3}$ trabaja en la idea de formular una metodología de monitoreo de los riesgos estratégicos, provenientes del contexto interno y externo de las organizaciones, que sirva como instrumento de vigilancia y proporcione información que permita administrar los riesgos estratégicos y mejorar la gestión empresarial.

Con el diseño de esta metodología, las empresas podrán tener una herramienta con la cual estén atentos a su desempeño y a su entorno, al analizar y estudiar los diferentes eventos adversos a sus propósitos, para extraer información importante que les permita tomar decisiones con mayor claridad, adicional a esto, podrán visualizar diferentes escenarios futuros a los que se podrían enfrentar, lo que genera en las compañías mayor maniobrabilidad y facilidad para superar obstáculos. 
Se espera diseñar un mecanismo dinámico que registre eventos en tiempo real y que permita tomar decisiones con datos actualizados y oportunos, que fomente una actitud proactiva frente a los riesgos estratégicos para la mejora de la gestión empresarial.

\section{DESARROLLO DEL TEMA Planteamiento del problema}

Según Alfonso Vélez, el riesgo, el cambio y la incertidumbre en las empresas son temas que tienen mayor fuerza a la hora de tomar decisiones; anteriormente las organizaciones tenían más control de las variables que intervenían en sus decisiones, al existir productos únicos, sin gran competencia, los gustos de los consumidores no podían variar porque se debían ajustar a la escasa oferta del mercado y por ende las compañías tenían claramente identificado a que se enfrentarían, debido a la estabilidad de la mayoría de las variables de su entorno, lo que les permitía realizar planes con mayor certeza. Los cambios de la humanidad y la evolución de las economías, hicieron que las organizaciones enfocaran su atención en las variables del ambiente que los afecta, debido a que estas influencian en gran medida la forma en que se debe comportar una empresa, si el ambiente cambia -y cada vez lo hace con mayor rapidez-, los niveles de incertidumbre aumentan y el modo en que opera una organización se verá afectado. De ahí que se empieza a pensar en la manera de disminuir estos grados de incertidumbre, porque los negocios operan normalmente bajo supuestos de producción, ventas, costos, que se toman como ciertos cuando se hacen las proyecciones; por lo que es necesario analizar las fuentes de agitación, que son las que alteran las condiciones en las que se desempeñan las compañías, porque de no hacerlo, e ignorar la inestabilidad del entorno, conduce a que la empresa, como sistema, se desoriente y deje de funcionar adecuadamente ${ }^{4}$.

Por lo anterior, en las organizaciones actuales se presentan grandes dificultades, por los riesgos que todos los días acechan a las compañías, escenarios cada vez más turbulentos, competidores más agresivos, gustos de los consumidores más volátiles, situaciones climáticas inestables, condiciones económicas dinámicas, etc., son variables que afectan de forma directa a las empresas y que se convierten en grandes barreras para la sostenibilidad de sus negocios. Es por esto,

${ }^{4}$ Alfonso Vélez, entrevista personal por Rubi Consuelo Mejía Quijano, Medellín, Julio de 2013 que el estudio y manejo de los riesgos estratégicos en las empresas se vuelve cada vez más importante y se convierte en un factor de ventaja competitiva para las mismas.

\section{Estado del arte}

Según Francisco Trullenque son pocas las organizaciones que gestionan sus riesgos en forma sistemática y completa, la gran mayoría de ellas estudia sólo con los riesgos de las actividades, es decir los operativos y son pocas las organizaciones que consideran los riesgos que pueden afectar sus lineamientos estratégicos y su posición competitiva (Trullenque, 2003:13).

Este mismo autor define el riesgo estratégico como "aquél que tiene un impacto destacado sobre las palancas básicas de creación de valor de la organización" (Trullenque, 2003:12). De otro lado, Deloitte lo define como "posibilidad de que un evento o acción, interna o externa, afecte adversamente la capacidad de una organización para ejecutar exitosamente sus estrategias y alcanzar los objetivos" (Deloitte \& Touche e IMEF, 2003: 2).

Algunos estudiosos del tema reconocen diversos riesgos estratégicos, tales como los generados por diferentes tipos de conducta, en los líderes organizacionales, que inducen al fracaso de la compañía, por ejemplo plantear metas inalcanzables para la empresa, negando allí la existencia del riesgo, dándole poca importancia a las posibles dificultades que se pueden presentar (Collins, 2010). Otros académicos promulgan la importancia de identificar oportunidades en los actuales ambientes hostiles, en las constantes amenazas inmersas en los contextos en los que se desempeñan las empresas, para poder crecer y lograr sostenimiento (Slywotzky \& Weber, 2008).

Conscientes de la necesidad que tienen las empresas de disminuir la incertidumbre y tomar decisiones mejor argumentadas teniendo presente los niveles de riesgos, la Universidad EAFIT ha realizado varias investigaciones en el campo de la administración de riesgos para brindar herramientas a las empresas que les permitan sortear con mayor facilidad estos escenarios a los que se enfrentan; inicialmente se participó en el diseño el Modelo Estándar de Control Interno para Entidades del Estado. MECI:1000, 2005, que involucra la administración de riesgos como uno de los Componentes del Sistema de Control; posterior- 
mente se creó el método Risicar (2006) y el Software Risicar (2008, 2010), los cuales tienen como objetivo contribuir a la implementación de la administración de riesgos.

Adicional a esto, en el año 2011 se creó la metodología para identificar los riesgos en los procesos de planeación estratégica (Prest), aplicable a cualquier tipo de empresa (pequeña, mediana, grande), para generar conciencia de la importancia que tiene la administración de los riesgos estratégicos para todas las organizaciones y los beneficios de su identificación y control. Estos modelos fueron diseñados teniendo presente los estándares y normas nacionales e internacionales que rigen el tema de Administración de riesgos e incluyen elementos para su monitoreo.

En el campo de la administración de riesgos y específicamente a través de los estándares y normas que la rigen, se ha definido el monitoreo como un componente importante, pero no se ha profundizado suficientemente en la manera como se diseñan los mecanismos de monitoreo y en especial de los riesgos estratégicos.

De acuerdo con el Estándar AS/NZ 4360:1999 uno de los primeros en el tema, el monitoreo se realiza con el objetivo de revisar la manera en que se está aplicando el sistema de administración de riesgos en las organizaciones y de identificar los cambios que puedan afectarlo en gran medida. Asimismo se considera una etapa importante porque revisa la efectividad del plan de tratamiento y estrategias de la organización, detectando los riesgos a los que se enfrenta.

Por su parte el Modelo de Control COSO del 2004 destaca la etapa de monitoreo, como una fase que garantiza la continuidad del proceso de administración de riesgos en las entidades, a través de la cual pueden detectarse las condiciones cambiantes que hay al interior y exterior de las organizaciones, para garantizar evaluaciones basadas en la realidad organizacional y tomar las medidas apropiadas para reducir los peligros a los que se ven expuestos.

El Modelo Estándar de Control Interno para el Estado Colombiano MECI 1000:2005, referencia la autoeva- luación como el mecanismo fundamental para llevar a cabo las funciones del monitoreo ante los factores críticos de éxito de los procesos y la detección de las desviaciones y cambios presentes continuamente en las empresas, tanto a nivel interno como externo, que pueden afectar la continuidad organizacional.

La Norma ISO 31000 del 2009 plantea el monitoreo y revisión, como una etapa indispensable para garantizar la eficacia del proceso de gestión de riesgos en las organizaciones, a través de indicadores, revisiones de los planes, políticas y marco de la gestión de riesgos, así como la detección de los cambios presentes en el entorno interno y externo de las empresas, y la identificación de riesgos emergentes en el proceso de implementación de medidas de tratamiento de los riesgos estratégicos principalmente. (ISO, 2009)

El COSO 2010, resalta la importancia de los eventos causa raíz, que con el pasar del tiempo se convierten en un evento intermedio que en última instancia conduce a un evento de riesgo; por lo anterior para identificar los indicadores clave de riesgo resulta útil pensar en la cadena de eventos que pueden conducir al suceso de riesgo, es decir, detectar las causas de ellos. (Beasley, Branson, Hancock, 2010:3)

\section{Marco conceptual}

El marco teórico que rige la investigación está conformado por los conceptos empresariales de riesgo estratégico, tipos de riesgo estratégico y monitoreo estratégico.

El riesgo estratégico se define como "la posibilidad de ocurrencia de un evento que pueda afectar la misión, visión, estrategias, objetivos y demás lineamientos organizacionales de alto nivel, en términos de amenazas que deben reducirse, contrarrestarse, o transformarse en oportunidades que impliquen beneficios para la organización"(Mejia, 2013:244).

Los tipos de riesgos estratégicos según la metodología de identificación de riesgos estratégicos Prest ${ }^{5}$ (Mejia, 2013) se dividen en internos y externos ${ }^{6}$. Los riesgos originados al interior de la organización pueden ser el riesgo de modelo de negocio inadecuado debido a que este está expuesto a la obsolescencia

\footnotetext{
${ }^{5}$ Desarrollada en la Universidad EAFIT por: Nuñez, M. A., Torres, M., Arboleda, O. L., y Mejia, R. C.

${ }^{6}$ Las cinco fuerzas de Porter es una herramienta que permite realizar un análisis de variables externas de una empresa. (Porter, 1991:1025)
} 
(Deloitte \& Touche, 2003: 128), riesgo de crecimiento desbordado que lleva a veces a las empresa a bajar los estándares para la selección del personal, debido a la urgencia de las contrataciones, los empleados nuevos pueden no poseer las destrezas y entrenamiento adecuado (Simons, 1999:19), riesgo de asignación ineficiente de recursos "ocurren durante las fases de captura de información, evaluación, toma de decisiones, y ejecución de programas, proyectos y oportunidades de negocio, ya sea en forma individual o con el concurso de terceros" (Bravo y Sanchéz, 2009:22), riesgo de gestión del talento humano, se puede presentar de distintas formas: inadecuada gestión del talento humano, lo que podría implicar prescindir de empleados con conocimientos y perfiles convenientes para la organización (Pascual, 2008:47), entre otros.

Los riesgos originados por el entorno externo organizacional pueden ser, entre otros, el riesgo de cambio en gustos y prioridades de los clientes debido a que "son personas impredecibles, irracionales, emotivas, curiosas y muy propensas al cambio. Los clientes no pueden permanecer quietos. Cambian de segmento; pasan de compradores de productos a compradores de valor o a compradores de precio, y de allí nuevamente al comienzo. Sus prioridades cambian de la calidad al precio" (Slywotzky, 2008:83), el riesgo de estancamiento que consiste en que las organizaciones dejan de crecer en términos de ventas, una de sus consecuencias es la disminución en el precio de sus acciones (Kotler y Caslione, 2010:19), riesgo de competidores, consistente en no identificar la competencia o prestarle poca atención; tener conocimiento de la competencia es un factor clave para alcanzar el éxito en una empresa, de lo contrario no se tomarían medidas para neutralizarla (Slywotzky, 2008:18).

De otro lado, el monitoreo estratégico se encarga de "evaluar ciertos factores, actividades y sus interrelaciones, de forma continua en periodos determinados, con el fin de identificar los efectos, las tendencias y los riesgos" (Gutiérrez, Sanchez, Piñeres y Yoshioka, 2005:198) que pueden afectar el cumplimiento de los objetivos estratégicos.

\section{METODOLOGÍA}

La investigación tiene un enfoque cualitativo, exploratorio y propositivo. El enfoque cualitativo, obedece a una investigación que no ofrece un análisis estadístico o algún tipo de cuantificación de los datos analizados, y busca responder preguntas como: ¿por qué?, ¿cómo? y ¿en qué forma?’(López y Salas, 2009:134) se están llevando a cabo las diferentes prácticas de monitoreo de riesgos estratégicos en las organizaciones.

Asimismo el carácter exploratorio de la investigación denota el interés por indagar un tema poco estudiado y conocer desde diferentes perspectivas y disciplinas, las opciones con las que cuentan las empresas para llevar a cabo la labor de monitoreo de los riesgos estratégicos.

La recolección de información se realiza a través de revisión documental, adicionalmente se utilizan entrevistas abiertas y semiestructuradas a profesionales con práctica en el monitoreo de riesgos estratégicos y a expertos conocedores del tema. Con la información obtenida se desarrolla el carácter propositivo de la investigación, al plantear una metodología para realizar el monitoreo de los riesgos estratégicos provenientes del ambiente interno y del entorno organizacional.

\section{AVANCES DE LA INVESTIGACIÓN}

De acuerdo con los objetivos de la investigación, se caracterizaron los riesgos estratégicos identificados inicialmente en la Metodología Prest mencionada anteriormente, y los adicionales que se identificaron en esta investigación.

Se reconocieron las prácticas de monitoreo interno y externo en la organización como la vigilancia tecnológica, la inteligencia competitiva, la inteligencia de negocios, los sistemas de alerta temprana, el diagnóstico organizacional, el cuadro de mando integral, inteligencia de mercados, que poseen herramientas para dar seguimiento a diferentes variables de interés para la organización. A continuación se da una breve explicación del papel de cada una de ellas.

\section{Prácticas de monitoreo}

\section{- Sistemas de alertas tempranas (SAT)}

Los sistemas de alerta temprana se conforman por varios instrumentos de apoyo al logro de los objetivos de una compañía, identifican cambios del entorno, tendencias que están en sus primeras fases y demás variables que pueden tener efectos importantes en las organizaciones. (Comai, 2011:492)

Además de esto se puede considerar que los indicadores de alerta temprana representan cuantitativa y cualitativamente información por medio de la cual 
se pueden establecer oportunidades y amenazas en sus primeros momentos antes de que se materialicen (Labaš, 2012:151-152); este sistema necesita de una actualización continua de datos para aumentar su efectividad y adicional a esto se debe realizar un registro de eventos anteriores que se convertirán en experiencia fundamental de esta herramienta, es un tema complejo porque el futuro es incierto, pero se hace necesario su estudio para saber afrontar las posibles situaciones venideras. (Krstevska, 2012:9)

\section{- Inteligencia Económica (IE)}

Las empresas tienen la necesidad de saber el rumbo de la economía en la que se encuentran para poder tomar decisiones correctas en el momento adecuado, es por eso que "varios bancos centrales y agencias económicas del mundo cuentan con indicadores líderes de actividad económica. Su seguimiento puede hacerse de manera desagregada, como en el caso del Business Cycle Dating Committee, del National Bureau of Economic Research (NBER), o combinando las variables consideradas líderes a través de un solo índice compuesto."(Kamil, Pulido, y Torres, 2010:498-499)

La inteligencia económica es definida por algunos autores como "un método que utiliza técnicas de vanguardia para aprender a conocer el medio en el que hay que actuar, identificar los socios con los que hay que competir, anticipar las amenazas que pueden aparecer para protegerse de ellas y negociar con posibilidades reales de éxito."(Juillet, 2006:135)

\section{- Vigilancia tecnológica e inteligencia com petitiva (IC)}

La inteligencia competitiva se define como el "proceso analítico que transforma los datos desagregados obtenidos de los competidores en conocimiento relevante, fiable y útil sobre los objetivos, recursos, capacidades y resultados de los competidores."(Múnera y Rodríguez, 2013:154).

La IC se compone de diferentes etapas "cada fase tiene características clave que influyen en la eficacia del sistema de inteligencia. En la primera es importante la existencia de procedimientos para inventariar y actualizar las necesidades tácticas y estratégicas. Para recoger y organizar información es importante identificar las fuentes adecuadas, y disponer de sistemas para su organización y almacenamiento."(García, Ortoll, y López, 2011:4)
"Conocer el entorno, identificar tendencias (nuevos temas, nuevos actores, nuevos productos, nuevos procesos, etc.), riesgos, oportunidades, etc., y actuar en consecuencia, son los objetivos básicos de la vigilancia tecnológica y la inteligencia competitiva. Además son procesos estrechamente unidos y orientados a la mejora de la competitividad de las empresas.”(Giménez y Román, 2001:3).

\section{- Inteligencia de negocios}

"Tiene por objetivo proporcionar la información sobre el entorno organizacional, identificar las amenazas y oportunidades, evitar las sorpresas desagradables, mejorar la planificación, aumentar la probabilidad de tomar buenas decisiones, reducir el riesgo organizacional y personal o, al menos, evaluar las opciones y las previsiones del riesgo/beneficio". (Barndt, 1994) Permite no sólo entender la competencia directa e indirecta, los comportamientos de los productos, las capacidades, el desempeño, la posición y las opciones de los clientes, de los proveedores y de los competidores, sino también los ámbitos tecnológico, comercial, político, económico, entre otros, al tiempo que incorpora el monitoreo del entorno con efectos actuales y futuros respecto de las decisiones empresariales (Cardona, 2005:21)

\section{- Cuadro de mando integral o Balanced Sco recard}

"Es un sistema de gestión desarrollado en los años noventa por Robert Kaplan y David Norton (The balanced scorecard, MIT Press, 1996), que permite expresar la estrategia de una organización desde cuatro perspectivas básicas accionistas, clientes, procesos internos y aprendizaje y crecimiento y mediante un conjunto de medidas (indicadores) de gran utilidad para controlar la ejecución de los planes" (Blanco, 2011:65). Este sistema ha sido ampliamente utilizado en todo tipo de compañías y ha contribuido a mejor el desempeño organizacional.

"Es una herramienta de aprendizaje organizativo. Los resultados que recogen los indicadores sirven para evaluar si hay que cambiar el modelo de negocio o incluso la estrategia. La comparación entre lo que se esperaba y lo que ocurre de verdad es una fuente de información útil para ajustar la forma de competir de la empresa" (Dávila, 1999: 39).

\section{- Diagnóstico organizacional}

Es fundamental realizar un análisis interno de la or- 
ganización para determinar el estado actual de la empresa, sus reales capacidades y necesidades, para lo cual se puede implementar el diagnostico organizacional que "es un proceso de comparación entre dos situaciones, la presente que hemos llegado a conocer a través de la indagación, y otra ya definida y supuestamente conocida que nos sirve de pauta o modelo". (Vidal, 2004:25)

\section{Metodología para monitorear los riesgos estraté- gicos}

La metodología propuesta para monitorear riesgos estratégicos consta de cinco fases, la primera comprende una serie de pre-requisitos para llevar a cabo las siguientes etapas que son: Definición del mecanismo de monitoreo, recolección de información, análisis de la información y toma de decisiones

\section{- $\quad$ Pre-requisitos}

Para llegar a desarrollar una metodología de monitoreo de riesgos estratégicos es deseable que la empresa esté madura en la administración de riesgos y en la planeación estratégica, lo cual facilita su implementación.

Si esto no existe en la organización, se debe iniciar con un ejercicio de sensibilización en los diferentes niveles de la empresa para crear conciencia de la necesidad de manejar los riesgos en general, y los estratégicos en particular.

También debe comunicarse expresamente la intención, por parte de la alta dirección, de trabajar en el tema de riesgos, e involucrar y enfocar a los diferentes niveles jerárquicos para que trabajen con todos los empleados hacia un mismo objetivo ${ }^{7}$.

En este proceso inicial también se selecciona el grupo de trabajo, debido a que deben existir personas dedicadas a esta función en la compañía, para que se responsabilicen de los riesgos y la supervisión de los mismos, lo ideal es que sea un grupo multidisciplinario, conformado por personas de diferentes áreas de la organización, que permita tener criterios variados y complementarios para el análisis de los riesgos estra-

\footnotetext{
${ }^{7}$ Carlos Fernando Lega, entrevista personal por Rubi Consuelo Mejía Quijano, Medellín, julio de 2013

${ }^{8}$ Emiliano Salazar, entrevista personal por Marcela Lega Ruiz, Medellín, agosto de 2013
}

tégicos, este grupo recibe un proceso de capacitación para garantizar que cuentan con los conocimientos necesarios en el tema de riesgos estratégicos, que les permita desempeñar las funciones requeridas por la metodología que se va a implementar ${ }^{8}$.

Un insumo vital para iniciar con la metodología del monitoreo de riesgos estratégicos es su identificación; debido a que una forma efectiva de empezar es tomar el top 5-10 de los riesgos más significativos a los cuales la organización se enfrenta, esto le permite a la empresa focalizar los esfuerzos para ser más efectivos en dicho monitoreo. (Beasley, Branson, Hancock, 2010:6).

Para identificar los riesgos estratégicos se puede recurrir a una gran variedad de metodologías o herramientas desarrolladas en diferentes disciplinas y propuestas en los estándares de riesgos ${ }^{9}$. Para evaluar los riesgos identificados, se analiza su probabilidad de ocurrencia y el impacto que generan, variables que se califican y dado el resultado permiten evaluar su gravedad. Normalmente los riesgos evaluados como graves o inaceptables son los seleccionados para ser monitoreados ${ }^{10}$.

Existen otras alternativas adicionales para definir los riesgos estratégicos a ser monitoreados como el analizar el registro de eventos de la compañía, que consiste en una bitácora de los riesgos ocurridos y que han impactado estratégicamente a la empresa, o empresas del sector; o a través de una autoevaluación que realice el grupo de dirección sobre las situaciones que normalmente consideran que "no dejan dormir al gerente", o por medio de otras metodologías que implemente la organización para evaluar sus riesgos.

En esta fase, también se debe analizar la cantidad de recursos disponibles en la organización, que garanticen la sostenibilidad de la metodología en el tiempo, es importante tener consciencia de la necesidad de invertir recursos tales como personal, herramientas, tiempo y dinero, y de la importancia de que sea una

\footnotetext{
${ }^{9}$ Se sugiere consultar la Norma ISO 31010 que ofrece la descripción, uso, beneficio y dificultades de una gran variedad de técnicas de identificación de riegos (ISO 31010, 2009) y el Libro Identificación de riesgos en el cual se presentan doce alternativas para identificar riesgos, incluidas algunas específicas para los riesgos estratégicos como Prest, Escenarios y Entrevistas. (Mejia 2013).

${ }^{10}$ Puede consultarse la norma ISO 31000 y el método Risicar que establece la forma como se califican y evalúan los riesgos (Mejia, 2006)
} 
implementación continua, para obtener resultados que generen valor para la organización y para la toma de decisiones.

\section{- $\quad$ Primera fase. Definición del mecanismo de monitoreo}

Su finalidad es construir el mecanismo de monitoreo de los riesgos priorizados que empleará la empresa y que le permitirá constituirse en un dispositivo de alerta temprana que le permitirá a la organización tener una actitud proactiva frente al riesgo.

En esta etapa se determina la manera en que se van a monitorear los riesgos estratégicos, que incluye el establecer los indicadores clave de riesgo, los cuales indican la presencia potencial, nivel o tendencia de un riesgo, y proporcionan información acerca de, si un riesgo se ha materializado o está surgiendo. Con ellos se puede observar el nivel de la exposición al riesgo y su tendencia, por lo cual se utilizan como orientadores de la acción a seguir y como señales de alerta temprana (Fraser y Simkins, 2010:126).

Los indicadores clave de riesgo incluyen: nombre del indicador, fórmula, meta del indicador, alcance geográfico, periodicidad, fuentes de información, medios de acceso a las fuentes de información, cadenas de eventos, nivel de tolerancia (estableciendo aquí los umbrales para generar señales de alerta temprana) y el responsable del riesgo, llamado también propietario del riesgo según la norma ISO 31000.

Otra manera de realizar el monitoreo de riesgos estratégicos, propuesta para los riesgos que no se pueden monitorear con indicadores, es el establecimiento de sesiones de autoevaluación, en las cuales se determina, a través de unos criterios predefinidos, una calificación que representa la gravedad del riesgo; para ello se debe disponer de información cualitativa o cuantitativa que soporte esta evaluación. La periodicidad de este mecanismo de autoevaluación depende de los niveles de exposición a los riesgos estratégicos definidos.

También se puede usar la técnica de escenarios para simular situaciones y validar que tanto las estrategias definidas por la compañía pueden verse afectadas, estos escenarios pueden variar periódicamente dados los cambios del entorno que afecten el desempeño de la empresa.
El registro de eventos puede ofrecer información relevante, al realizar un análisis histórico de los acontecimientos adversos de la compañía y posteriormente efectuar un análisis de tendencias.

\section{- $\quad$ Segunda fase. Recolección de información}

Una vez seleccionados los riesgos que se van a monitorear, y definido el mecanismo de monitoreo, se establecen las necesidades de información que tiene la empresa, respecto a las variables que condicionan la posibilidad de ocurrencia de los riesgos estratégicos sujetos al monitoreo. También se realiza un estudio interno donde se identifica, si de alguna manera o en algún área se recopila esta información y se establece donde la obtienen, cómo la utilizan y cómo la documentan.

En esta fase de la metodología para monitorear los riesgos estratégicos, se determina la estrategia de búsqueda y los responsables, si la búsqueda de información se va a realizar por medio de terceros, personal interno o mixto, dejando claro las funciones de cada persona.

Dentro de esta estrategia se seleccionan los tipos de fuentes para acceder a la información que pueden ser internas (datos impresos, bases de datos electrónicas, intranet, informes y balances) o externas (datos impresos, bases de datos electrónicas, diarios, revistas, radio, televisión, fuentes informales, entrevistas personales, reuniones, fuentes locales y nacionales). "para cada eje temático se definen las fuentes, la exhaustividad de las mismas, se establecen las palabras claves (en distintos idiomas) y se construyen las ecuaciones de busqueda" (AECID, 2012:37)

Dentro de esta segunda fase, una vez se obtiene la información se procede con el registro de lo recolectado (físico o digital) y la organización (selección de datos relevantes, validación de los datos, organización de la información según el tipo de objetivo).

\section{- Tercera fase. Análisis de la información}

La tercera fase es donde se realiza el análisis de la información, en la cual se comparan los resultados obtenidos en el proceso de monitoreo, con los objetivos iniciales o metas del comportamiento de los riesgos, establecidos en la primera fase de la metodología, teniendo presente que se debe "medir periódicamente el progreso frente al plan para la gestión del riesgo y las desviaciones con respecto a este" (Icontec, 2011:16); 
luego se establece una relación de indicadores clave de riesgo, y se organiza toda la información para generar un modelo coherente del entorno futuro de negocios al que se puede enfrentar la organización, posterior a esto se piensa en distintos escenarios posibles para discutir los efectos que tendrían en las estrategias actuales de la compañía, para determinar otras opciones de estrategias, adicionalmente se detectan oportunidades, amenazas, fortalezas y debilidades, lo que hace visible los posibles riesgos futuros de la empresa y las áreas que requieren mayor comprensión y conocimiento. (Juut y Winther, 2010:160-161)

Adicionalmente se identifican las fallas de los controles actuales que realiza la organización para manejar los riesgos estratégicos y se definen las medidas necesarias para su solución; después de todo lo anterior, se crean nuevos indicadores clave de riesgo, esto porque el entorno es volátil y el monitoreo de los riesgos debe ser dinámico para su máximo aprovechamiento.

En la parte final de esta fase se elabora una recopilación de todos los análisis, derivados de la información obtenida durante todo el proceso de monitoreo de riesgos estratégicos, y se elabora el informe que será presentado a la alta dirección.

\section{- Cuarta fase. Toma de decisiones}

En la cuarta fase y última de la metodología, se toman decisiones como fruto de la discusión que genera en el grupo directivo de la empresa la información (analizada y recopilada en el informe elaborado en la fase anterior).

Las personas encargadas del direccionamiento de la compañía tienen los elementos necesarios para definir qué medidas se deben implementar (estrategias emergentes, contingentes y/o eliminación de estrategias).

En esta fase también se realiza la evaluación del desempeño de todo el proceso realizado y se originan comentarios de mejoramiento y retroalimentación.

Todo esto con el objetivo de que la compañía pueda responder oportuna y adecuadamente a los riesgos estratégicos que pueden afectarlas.

\section{CONCLUSIONES}

La clara necesidad por parte de las empresas de tener un sistema que les permita monitorear su entorno y realizar análisis que les facilite la detección de riesgos estratégicos, hace necesarios este tipo de estudio, que orienta a las organizaciones actuales para que sean entidades proactivas ante el riesgo y con pensamiento a futuro; lo que les permite ajustar sus planes de acuerdo con los cambios del entorno, y las capacita para enfrentarse a escenarios volátiles.

Para aplicar la metodología propuesta, la organización debe sensibilizar a los empleados en la importancia de manejar y monitorear los riesgos estratégicos, al igual que identificar, evaluar y priorización estos riesgos, establecer un mecanismo de monitoreo, determinar cómo se hará la búsqueda de información y cómo se establecerá su validez. Con el análisis de la información recopilada se pueden tomar acciones sustentadas para eliminar estrategias, reformularlas o definir estrategias contingentes, que respondan a riesgos no identificados y que pueden materializarse poniendo en peligro la empresa; con el fin de mejorar el desempeño organizacional y garantizar su supervivencia. 


\section{BIBLIOGRAFIA}

- AECID y otros, (2012). Guía metodológica de práctica de la vigilancia tecnológica e inteligencia competitiva. Valencia y Medellín

- Barndt, W. (1994). User-directed competitive intelligence: closing the gap between supply and demand. London: Quorum Books.

- Beasley, M., Branson, B. y Hancock, B. (2010). Developing key risk indicators to strengthen enterprise risk management:-How key risk indicators can sharpen focus on emerging risk. New York: Committee of Sponsoring Organizations of the Treadway Commission (COSO)

- Blanco, E. (2011). “Una herramienta efectiva, pero exigente”. En Debates IESA, 16(3), 65-68

- Bravo, O. y Sanchéz, M. (2009). Gestión integral de Riesgos. Bogotá: Bravo \& Sánchez

- Cardona, S. (2005). La inteligencia de negocios y su aplicación en algunas empresas del área metropolitana de Medellín. Tesis de maestría en administración, Universidad EAFIT, Medellín.

- Collins, J. (2010). Cómo caen los poderosos: y por qué algunas compañías nunca se rinden. Bogotá: Grupo editorial Norma

- Comai, A. (2011). "Inteligencia competitiva: Logros y desafíos". En El profesional de la información, 20(5), 489-493

- COSO (Committee of Sponsoring Organizations Of The Treadway Commission). (2004). Gestión de riesgos corporativos - Marco integrado. Técnicas de aplicación, Jersey City: Permissions Editor.

- Dávila, A. (1999). "Nuevas herramientas de control: El cuadro de mando integral”. En Revista de antiguos alumnos, Septiembre, 34-40

- Deloitte \& Touche e IMEF. (2003). Administración integral de los riesgos de negocio. México: Instituto Mexicano de Ejecutivos de Finanzas.

- Fraser, J. y Simkins, B. (2010). Enterprise Risk Management: Today's Leading Research and Best Practices for Tomorrow's Executives. New Jersey: John Wiley \& Sons

- Garcia, M., Ortoll, E. y López, A. (2011). "Aplicaciones emergentes de inteligencia competitiva en las universidades". En El profesional de la información, 20(5), 503-509.

- Giménez, E. y Román, A. (2001). "Vigilancia tecnológica e inteligencia competitiva: conceptos, profesionales, servicios y fuentes de información”. En El profesional de la información, 10(5), 11-20.

- Gutiérrez, V., Sánchez, M., Piñeres, J. y Yoshioka, A. (2005). "Diseño de un sistema de monitoreo para el cluster bioindustrial del occidente Colombiano". En Cuadernos de Administración, 18(30)

- Icontec Internacional. (2011). NTC-ISO 31000. Norma técnica colombina. Gestión del riesgo. Principios y directrices, Bogotá, Icontec

- ISO (international organization for standardization)31000. (2009).Risk management-principles and guidelines, Suiza,

- Juillet, A. (2006). "Principios y aplicación de la inteligencia económica". En Inteligencia y seguridad: Revista de análisis y prospectiva, 1, 113-136

- Juut, T. y Winther, P. (2010). Strategic Risk Management Practice. How to deal effectively with major corporate exposures. New York: Cambridge University Press

- Kamil, H., Pulido, J. D., \& Torres, J. L. (2010). "El IMACO: un índice mensual líder de la actividad económica en Colombia”. En Monetaria, 33(4), 495-548

- Kaplan, R. \& Norton, D. (2008). The Execution Premium, Integrando la estrategia y las ope- 
raciones para lograr ventajas competitivas. España: Ediciones Deusto.

- Kotler, P. y Caslione, J. (2010). Caótica: Administración y Marketing en tiempos de caos. Colombia: Grupo Editorial Norma.

- Krstevska, A. (2012). "Early Warning Systems: Testing in Practice”. En IUP Journal of Financial Risk Management, 9(2), 7-22.

- Labaš, D. (2012). Strategic Early Warning Systems And Information Technology Support In Croatian Firms. Conference Proceedings: International Conference of the Faculty of Economics Sarajevo (ICES), 150-167.

- López, F. y Salas, H. (2009). "Investigación cualitativa en Administración”. En Revista de Epistemología de Ciencias Sociales, Cinta de Moebio, 35

- Mejía, R. (2013). Identificación de riesgos. Medellín: Fondo Editorial Universidad EAFIT.

- Munera, J. y Rodríguez, A. (2013). Estrategias de Marketing, Un enfoque basado en el proceso de dirección. España: ESIC editorial

- Pascual, R. (2008). "Nuevos retos de la gerencia de riesgos: un modelo de gestión y retención del talento", En Gerencia de riesgos y seguros, 25(101), 38-47

- Porter, M. (1991). La ventaja competitiva de las naciones. Buenos Aires: Editorial Vergara.

- Simons, R. (1999). "Qué tan riesgosa es su empresa”. En Dinero, 64, 80-92

- Slywotzky, A. y Weber, K. (2008). Riesgo positivo: Cómo convertir las grandes amenazas en oportunidades. Colombia: Grupo Editorial Norma.

- Trullenque, F. (2003). “Gestión estratégica del riesgo: Cómo gestionar la incertidumbre”. En Estrategia Financiera, 25(193), 12-18

- Vidal, E. (2004). Diagnostico Organizacional. Evaluación sistémica del desempeño empresarial en la era digital. Bogotá: Ecoe Ediciones. 